\title{
Formation of the First Supermassive Black Holes
}

\section{Citation}

Bromm, Volker, and Abraham Loeb. 2003. "Formation of the First Supermassive Black Holes." The Astrophysical Journal 596 (1): 34-46. https://doi.org/10.1086/377529.

\section{Permanent link}

http://nrs.harvard.edu/urn-3:HUL.InstRepos:41393341

\section{Terms of Use}

This article was downloaded from Harvard University's DASH repository, and is made available under the terms and conditions applicable to Other Posted Material, as set forth at http:// nrs.harvard.edu/urn-3:HUL.InstRepos:dash.current.terms-of-use\#LAA

\section{Share Your Story}

The Harvard community has made this article openly available.

Please share how this access benefits you. Submit a story.

Accessibility 


\title{
FORMATION OF THE FIRST SUPERMASSIVE BLACK HOLES
}

\author{
Volker Bromm $^{1}$ and Abraham Loeb ${ }^{1,2,3}$ \\ Received 2002 December 18; accepted 2003 June 16
}

\begin{abstract}
We consider the physical conditions under which supermassive black holes could have formed inside the first galaxies. Our smoothed particle hydrodynamics simulations indicate that metal-free galaxies with a virial temperature of $\sim 10^{4} \mathrm{~K}$ and suppressed $\mathrm{H}_{2}$ formation (due to an intergalactic UV background) tend to form a binary black hole system that contains a substantial fraction $(\gtrsim 10 \%)$ of the total baryonic mass of the host galaxy. Fragmentation into stars is suppressed without substantial $\mathrm{H}_{2}$ cooling. Our simulations follow the condensation of $\sim 5 \times 10^{6} M_{\odot}$ around the two centers of the binary down to a scale of $\lesssim 0.1 \mathrm{pc}$. Low-spin galaxies form a single black hole instead. These early black holes lead to quasar activity before the epoch of reionization. Primordial black hole binaries lead to gravitational radiation emission at redshifts $z \gtrsim 10$ that would be detectable by Laser Interferometer Space Antenna.

Subject headings: black hole physics — cosmology: theory — galaxies: formation — hydrodynamics — quasars: general

On-line material: color figures
\end{abstract}

\section{INTRODUCTION}

Supermassive black holes (SMBHs) are believed to provide the power source of quasars via the accretion of surrounding gas (Salpeter 1964; Zeldovich 1964; Lynden-Bell 1969; Rees 1984). They were able to form already at early cosmic times, as implied by the recent discovery of quasars at redshifts $z \gtrsim 6$ (Becker et al. 2001; Djorgovski et al. 2001; Fan et al. 2002). The existence of SMBHs with inferred masses of $\gtrsim 10^{9} M_{\odot}$, less than a billion years after the big bang, provides important constraints on any formation scenario (Haiman \& Loeb 2001).

The gas physics involved in the formation of SMBHs is still not well understood (Rees 1984, 2002; Loeb \& Rasio 1994; Barkana \& Loeb 2001). In this work, we attempt to simulate this process under the simplest and best-prescribed set of initial conditions, those dictated by the early universe. The earliest galaxies are simple in that they are the first condensations of gas to grow out of the seed inhomogeneities in the early universe. The composition of the primordial gas is determined by big bang nucleosynthesis (Burles, Nollett, \& Turner 2001 and references therein), and any primordial magnetic fields are not expected to be dynamically significant.

Previous numerical simulations of early luminous structures (Bromm, Coppi, \& Larson 1999, 2002; Abel, Bryan, \& Norman 2000, 2002; Nakamura \& Umemura 2001) have focused on the formation of stars inside the very first gaseous objects with a mass of $\sim 10^{5} M_{\odot}$, just above the cosmological Jeans mass. Because of their low virial temperature (hundreds of kelvins), fragmentation into stars is possible inside these objects through the formation of molecular hydrogen, $\mathrm{H}_{2}$, which cools efficiently via rotational-vibrational transitions even at these low temperatures. However, a relatively modest UV flux is sufficient to photodissociate the fragile $\mathrm{H}_{2}$

\footnotetext{
${ }^{1}$ Astronomy Department, Harvard University, 60 Garden Street, Cambridge, MA 02138; vbromm@cfa.harvard.edu; loeb@ias.edu.

2 Institute for Advanced Study, Princeton, NJ 08540.

${ }^{3}$ Guggenheim Fellow.
}

molecules and suppress their role in cooling the gas (Haiman, Rees, \& Loeb 1997; Ciardi et al. 2000; Haiman, Abel, \& Rees 2000). A destructive flux of UV photons could be produced by a small, early population of stars.

Here we consider dwarf galaxies with virial temperatures $\gtrsim 10^{4} \mathrm{~K}$, inside of which atomic cooling is effective (Oh \& Haiman 2002). These galaxies comprise a substantial population at $z \sim 10$ (Barkana \& Loeb 2001) that is expected to dominate the reionization of hydrogen at $z \gtrsim 6$ (Wyithe \& Loeb 2003a). We use smoothed particle hydrodynamics (SPH) simulations to describe the cooling and dynamics of the gas for different choices of its total angular momentum (or spin parameter), following the suggestion by Eisenstein \& Loeb (1995) that low-spin systems would be more susceptible to the formation of an SMBH. If $\mathrm{H}_{2}$ cooling is suppressed inside these galaxies, then their gas will not cool below $10^{4} \mathrm{~K}$. When the temperature to which the gas cools is only somewhat lower than the virial temperature of the host galaxy (at which the pressure force balances gravity for the gas), we expect that fragmentation into small clumps will be avoided, and the gas will tend to condense isothermally (at its temperature floor of $\sim 10^{4} \mathrm{~K}$ ) into large clumps. Such large clumps may then collapse to form an SMBH, possibly through an intermediate stage of a supermassive star (SMS). The viability of this scenario relies on the suppression of molecular $\mathrm{H}_{2}$ cooling, which when present is capable of cooling the gas to a temperature as low as $200 \mathrm{~K}$. We therefore start our analysis in $\S 2$ by considering the cosmic UV background expected from the first stars. Later on, in $\S 5$ we carry out numerical simulations to determine the level of UV radiation necessary for the photodissociation of $\mathrm{H}_{2}$ molecules during the early collapse phase of the above dwarf galaxies.

The SPH code we use is the same as was used to simulate the formation of the first massive stars (Bromm et al. 1999, 2002), except that we apply it to more massive galaxies, where atomic cooling is effective. Our goal is to find whether there is a direct route to the formation of SMBHs under these circumstances. Although our focus is on gasdynamics, we note that the first massive black holes ( $\mathrm{BHs}$ ) may have 
also formed out of the gravitational dynamics of clusters of stellar-mass BHs (Larson 2000, 2002; Madau \& Rees 2001; Schneider et al. 2002; Islam, Taylor, \& Silk 2003; Volonteri, Haardt, \& Madau 2003).

Throughout this paper, we assume a standard $\Lambda \mathrm{CDM}$ cosmology, with a total density parameter in matter of $\Omega_{m}=1-\Omega_{\Lambda}=0.3$ and in baryons of $\Omega_{b}=0.045$. The Hubble constant is $h=H_{0} /\left(100 \mathrm{~km} \mathrm{~s}^{-1} \mathrm{Mpc}^{-1}\right)=0.7$, and the present-day power-spectrum amplitude is $\sigma_{8}=0.9$ in spheres of radius $8 h^{-1} \mathrm{Mpc}$.

\section{THE COSMOLOGICAL CONTEXT}

We first outline the basic physical reason why small protogalactic systems of virial temperature $\sim 10^{4} \mathrm{~K}$ and mass $\sim 10^{8} M_{\odot}$, collapsing before the epoch of reionization at redshifts $z \gtrsim 10$, provide intriguing sites for the formation of the first SMBHs.

The direct collapse of a primordial gas cloud into a central compact object is made difficult by fragmentation and consequent star formation (Loeb \& Rasio 1994). The ability of the gas to fragment depends on the presence of an efficient cooling mechanism. By a redshift of $z \sim 10$, the intergalactic medium (IGM) is expected to be enriched with heavy elements from the first generation of stars to a level of $Z \gtrsim 10^{-3.5} Z_{\odot}$ (see, e.g., Gnedin \& Ostriker 1997; Mackey, Bromm, \& Hernquist 2003). Recent numerical investigations have shown that such a metallicity is sufficient to enable the gas to efficiently cool, fragment, and subsequently form stars (Omukai 2000; Bromm et al. 2001a; Bromm \& Clarke 2002). It is plausible that in some regions of the IGM, the gas that collapses into a dwarf galaxy has not been enriched with metals in excess of $Z_{\text {crit }} \sim 10^{-3.5} Z_{\odot}$ (see, e.g., Thacker, Scannapieco, \& Davis 2002; Furlanetto $\&$ Loeb 2003). In this paper, we investigate the collapse of a virtually metal-free system of this type.

It is well known that molecular hydrogen can effectively cool metal-free gas and thus enable the formation of the first stars at $z \simeq 20-30$ (see, e.g., Haiman, Thoul, \& Loeb 1996; Tegmark et al. 1997). Even in the absence of metals, star formation is therefore expected to occur, and the subsequent negative feedback due to supernova (SN) explosions would prevent the assembly of large quantities of gas in the center of the shallow dark matter (DM) potential well that characterizes the first dwarf galaxies (see, e.g., Dekel \& Silk 1986). Molecular hydrogen, however, is fragile and can readily be destroyed by photons in the Lyman-Werner (LW) bands, within the energy range $11.2-13.6 \mathrm{eV}$, via the two-step Solomon process (Stecher \& Williams 1967),

$$
\mathrm{H}_{2}+\gamma \rightarrow \mathrm{H}_{2}^{*} \rightarrow 2 \mathrm{H} .
$$

The intermediate stage involves an excited electronic state, $\mathrm{H}_{2}^{*}$, from which a fraction of the subsequent decays end in the vibrational continuum of the ground state, resulting in the dissociation of the molecule.

The question then arises whether $\mathrm{H}_{2}$ cooling can indeed be suppressed in the pre-reionization dwarf galaxies considered in this paper. These systems are rather massive compared to the $\sim 10^{5}-10^{6} M_{\odot}$ halos that host the formation of the first stars at $z \gtrsim 20$. The gas might then be able to selfshield against the photodissociating LW background (see, e.g., Glover \& Brand 2001; Machacek, Bryan, \& Abel 2001). Close to the epoch of reionization, however, a signifi- cant flux in the LW bands $(h \nu<13.6 \mathrm{eV})$ is expected. To estimate the LW flux, we first consider the flux of ionizing radiation just above the Lyman limit,

$$
J_{\nu}^{+} \simeq \frac{h c}{4 \pi} \frac{N_{\gamma} X \rho_{b}(z=10)}{m_{\mathrm{H}}} .
$$

Here $\rho_{b}(z=10)$ is the baryonic density at $z \simeq 10, X=0.76$ the mass fraction in hydrogen, and $m_{\mathrm{H}}$ the mass of a hydrogen atom. We assume that $N_{\gamma} \sim 10$ ionizing photons per baryon are required to reionize the universe (Wyithe \& Loeb 2003a). Evaluating this expression for the cosmological parameters given above, we find $J_{21}^{+} \sim 40$. Here and in the remainder of the paper, UV fluxes are normalized as $J_{\nu}=J_{21} \times 10^{-21} \mathrm{ergs} \mathrm{cm}^{-2} \mathrm{~s}^{-1} \mathrm{~Hz}^{-1} \mathrm{sr}^{-1}$.

The flux in the LW bands just below the Lyman limit $J_{\nu}^{-}$ could, however, be much larger than $J_{\nu}^{+}$. Assuming that only a fraction, $f_{\text {esc }}$, of the ionizing photons can escape from the star-forming halos, we have $J_{21}^{-} \simeq J_{21}^{+} / f_{\text {esc }} \sim$ $4 \times 10^{3}\left(f_{\text {esc }} / 0.01\right)$, where $f_{\text {esc }}$ is expected to be low at high redshifts (Wood \& Loeb 2000 and references therein). ${ }^{4}$

The strong UV background flux estimated above makes it possible for $\mathrm{H}_{2}$ formation to be effectively suppressed close to the redshift of reionization. In the absence of molecular hydrogen, however, cooling can still proceed via atomic transitions in halos of mass

$$
M \gtrsim 10^{8} M_{\odot}\left(\frac{1+z}{10}\right)^{-3 / 2}
$$

(see Barkana \& Loeb 2001). The virial temperature, $T_{\text {vir }} \sim 10^{4} \mathrm{~K}$, in these more massive halos allows for the very efficient cooling of the gas via lines of atomic hydrogen. Notice that in this case where the gas temperature is close to the virial temperature, the gas cloud as a whole can undergo collapse, but it will not be able to fragment until high enough densities are reached so that the Jeans mass has declined sufficiently. A halo of total mass $\sim 10^{8} M_{\odot}$ and a collapse redshift $z \sim 10$ correspond to a $2 \sigma$ peak in the random field of primordial density fluctuations.

In $\S 4$, we describe numerical simulations of such a $2 \sigma$ peak. We consider the evolution with and without the presence of $\mathrm{H}_{2}$. To ascertain the importance of $\mathrm{H}_{2}$, we consider the limiting cases of a halo made of purely atomic hydrogen and of a halo where $\mathrm{H}_{2}$ is allowed to form without any negative feedback. Subsequently, in $\S 5$ we discuss simulations in which an external LW background is included. We assess, in particular, the critical LW flux required to prevent molecules from forming. This required background flux may then be compared to the available flux at $z \sim 10$, as estimated in this section.

\section{NUMERICAL METHODOLOGY}

\subsection{Large-Scale Simulations}

The evolution of the dark matter and gas components is calculated with a version of TREESPH (Hernquist \& Katz 1989), combining the SPH method with a hierarchical (tree)

\footnotetext{
${ }^{4}$ A strong background of photodissociating photons could also result from star formation in the dwarf galaxy itself (see, e.g., Omukai \& Nishi 1999; Oh \& Haiman 2002). However, this would also result in the production of metals, probably leading to the rapid enrichment of the galaxy beyond $Z_{\text {crit }}$.
} 
gravity solver (see Bromm et al. 2002 for further details). Here we briefly describe the additions to the code that are necessary for the investigation of zero-metallicity gas. These include a method to treat the radiative cooling of the gas, together with a multispecies chemical reaction network and a technique to create sink particles. The thermal evolution of the gas is governed by the equation

$$
\frac{D u}{D t}=\frac{P}{\rho^{2}} \frac{D \rho}{D t}+\frac{\Gamma-\Lambda}{\rho},
$$

where $D / D t$ is the Lagrangian time derivative, $P$ and $\rho$ are the gas pressure and density, respectively, $u$ is the specific internal energy (in ergs $g^{-1}$ ), and $\Gamma$ and $\Lambda$ are the contributions from radiative heating and cooling, respectively (in ergs $\mathrm{cm}^{-3} \mathrm{~s}^{-1}$ ). Since we are concerned with a system that formed prior to the epoch of reionization, we assume that there is no heating due to a photoionizing background $(\Gamma=0)$. Radiative cooling is due to lines of atomic hydrogen at gas temperatures $T \gtrsim 10^{4} \mathrm{~K}$ and to lines of molecular hydrogen at lower temperatures. We have implemented the $\mathrm{H}_{2}$ cooling function given by Galli \& Palla (1998). Finally, the first term on the right-hand side describes adiabatic cooling due to expansion or heating due to compression.

Since radiative cooling to temperatures below that of the cosmic microwave background $(\mathrm{CMB}), T_{\mathrm{CMB}}=$ $2.7 \mathrm{~K}(1+z)$, is not thermodynamically possible, we write the cooling term as

$$
\Lambda=\Lambda(T)-\Lambda\left(T_{\mathrm{CMB}}\right) .
$$

For $T<T_{\mathrm{CMB}}$, radiative cooling consequently turns into heating. This approximate treatment ensures that $T \geq$ $T_{\mathrm{CMB}}$, unless cooling proceeds via adiabatic expansion.

The chemical reaction network comprises the nine species $\mathrm{H}, \mathrm{H}^{+}, \mathrm{H}^{-}, \mathrm{H}_{2}, \mathrm{H}_{2}^{+}, e^{-}, \mathrm{He}, \mathrm{He}^{+}$, and $\mathrm{He}^{++}$, including the reactions given in Haiman et al. (1996). Our implicit, backward-differencing method to solve the coupled set of rate equations is fast and accurate (see Bromm et al. 2002 for test calculations).

We have devised an algorithm to merge SPH particles in high-density regions in order to overcome the otherwise prohibitive time step limitation, as enforced by the Courant stability criterion. To follow the simulation for a few dynamical times, we allow SPH particles to merge into more massive ones, provided they exceed a predetermined density threshold. More details of the merging algorithm are given in Bromm et al. (2002).

\subsection{Small-Scale Simulations}

The sink-particle technique allows one to study the collective dynamics of multiple centers of condensation, such as merging between clumps and ongoing accretion over many dynamical timescales (see, e.g., Bate, Bonnell, \& Price 1995; Bate, Bonnell, \& Bromm 2003). It is important, however, to also be able to follow the collapse to increasingly high density. To accomplish this, we have carried out a complementary simulation in which we do not create sink particles. Instead, we focus on the highest density region in one of the large-scale simulations, resample the density field with an increased number of SPH particles, and adopt a rapidly decreasing time step according to $\Delta t_{\text {sys }} \propto 1 /\left(G \rho_{\max }\right)^{1 / 2}$. Here $\rho_{\max }$ is the maximum gas density at a given instant, and $\Delta t_{\text {sys }}$, the system time step, is the maximum allowed time by which a particle is advanced within the multiple-time step scheme employed in the simulations (see Hernquist \& Katz 1989). Under these conditions, the overall system hardly evolves at all, while the runaway collapse of one small region proceeds on a sufficiently short timescale. The mass resolution of a simulation is approximately

$$
M_{\text {res }}=\left(\frac{N_{\text {neigh }}}{N_{\mathrm{SPH}}}\right) M_{b},
$$

where $N_{\text {neigh }} \simeq 32$ is the number of particles within a given $\mathrm{SPH}$ smoothing kernel, $N_{\mathrm{SPH}}$ the total number of SPH particles, and $M_{b}$ the total baryonic mass. To avoid numerical fragmentation, the Jeans scale has to be resolved (Bate \& Burkert 1997): $M_{\text {res }}<M_{\mathrm{J}}$. After the onset of gravitational instability in the large-scale simulation, the rapid increase in density leads to a violation of this criterion. At this point, a sink particle is created in the simulation.

In our refined simulation, we follow a different computational strategy (see also Bromm 2000). Our starting configuration is the large-scale simulation, which we stop a short time before the central region would otherwise undergo runaway collapse. We now apply the following resampling procedure to the fluid within this region. Every SPH particle in the original, unrefined simulation acts as a parent particle denoted by $p$ and spawns $N_{\text {ref }}$ child particles denoted by $k$, where $N_{\text {ref }}=50$ in this paper. The child particles are distributed according to the SPH smoothing kernel $W\left(\boldsymbol{r}_{k}-\boldsymbol{r}_{p} ; h_{p}\right)$, by employing a standard Monte Carlo comparison-rejection method (see, e.g., Press et al. 1992). Here $h_{p}$ is the smoothing length of the parent particle. The velocity, temperature, and fractional abundances of the nine species included in the reaction network are directly inherited from the parent particle, $\boldsymbol{v}_{k}=\boldsymbol{v}_{p}, \quad T_{k}=T_{p}$, and $y_{k}=x_{p}$ for all $k$, respectively. Finally, each child particle is assigned a mass of $m_{k}=m_{p} /$ $N_{\text {ref }}$. This procedure conserves linear and angular momentum well. Energy is also conserved well, although there arises a small artificial contribution to the gravitational potential energy due to the discreteness of the resampling. The resampling described in $\S 4.2$ results in $N_{\mathrm{SPH}}=65,500$ within the central high-resolution region, and the mass resolution is now $M_{\text {res }} \simeq 500 M_{\odot}$, as compared to $M_{\text {res }} \simeq$ $20,000 M_{\odot}$ in the original simulation.

As we follow our simulation to densities in excess of $n_{\mathrm{H}} \sim 10^{7} \mathrm{~cm}^{-3}$, opacity effects begin to modify the thermal behavior of the gas. For simplicity, we continue to use the optically thin rate for cooling due to the collisional excitation of atomic hydrogen lines (Cen 1992). While this is clearly an oversimplification, we have verified that the resulting thermal evolution of the gas is very similar to what is found in a more sophisticated treatment of the cooling processes at high densities (Omukai 2001).

Our technique of refining a coarser, parent simulation and following the further collapse with increased resolution is conceptually similar to the adaptive mesh refinement (AMR) method, which was originally developed by Berger \& Oliger (1984). The AMR method has already been successfully applied to astrophysical problems (see, e.g., Truelove et al. 1998; Norman \& Bryan 1999), but our approach is one of the first attempts of implementing such a scheme within SPH (see also Kitsionas \& Whitworth 2002). 


\subsection{External UV background}

To investigate the effect of an external UV background on the evolution of the gas, we have included the relevant photoreactions in our chemical network. The photorates (in units of $\mathrm{s}^{-1}$ ) are given by

$$
k_{\mathrm{ph}}=4 \pi \int_{\nu_{\mathrm{th}}}^{\infty} \frac{\sigma_{\nu} J_{\nu} d \nu}{h \nu},
$$

where $h \nu_{\text {th }}$ is the threshold energy for a specific reaction. The cross-sections are given in Haiman et al. (1996). Following Omukai (2001), we consider both a power-law spectrum, $J_{\nu} \propto \nu^{-1}$, appropriate for quasar sources, and a thermal Planck spectrum, $J_{\nu} \propto B_{\nu}\left(T_{*}\right)$, appropriate for stellar sources. In the latter case, we evaluate the photorates for two different values of the radiation temperature: $T_{*} \simeq 10^{4}$ and $10^{5} \mathrm{~K}$, corresponding to "normal" Population II stars and very massive Population III stars, respectively (Bromm, Kudritzki, \& Loeb 2001b). For each spectrum, we normalize the flux at the Lyman limit with a normalization constant $J_{21}$ and assume zero flux at $h \nu>13.6 \mathrm{eV}$ because of intergalactic $\mathrm{H}$ i absorption.

To take into account the effect of self-shielding, we write the $\mathrm{H}_{2}$ photodissociation rate as $k_{\text {diss }} \propto J_{21} f_{\text {shield }}$, with a proportionality constant that depends on the chosen spectrum. For the shielding factor, we use the approximate expression $f_{\text {shield }} \simeq \min \left[1,\left(N_{\mathrm{H}_{2}} / 10^{14} \mathrm{~cm}^{-2}\right)^{-0.75}\right]$ (Draine \& Bertoldi 1996). This formula is accurate only for a static medium, and it will grossly overestimate the $\mathrm{H}_{2}$ line opacity in the presence of large-scale velocity gradients. In our simulations, we encounter moderately strong bulk flows, with velocities on the order of the sound speed. We therefore conservatively overestimate the effect of self-shielding. The $\mathrm{H}_{2}$ column density is estimated from local quantities only as follows: $N_{\mathrm{H}_{2}} \simeq 0.1 f_{\mathrm{H}_{2}} n_{\mathrm{H}} L_{\text {char }}$. We define a local characteristic length, $L_{\text {char }}$, such that the total baryonic mass would be contained in a sphere of uniform density, $n_{\mathrm{H}}$, and radius, $L_{\text {char. }}$. Thus, we have

$$
L_{\text {char }}=\left(\frac{3 X M_{b}}{4 \pi m_{\mathrm{H}} n_{\mathrm{H}}}\right)^{1 / 3} .
$$

By comparing with the column densities that are actually realized in the simulations, we have verified the validity of this prescription.

We neglect any contributions to the heating of the gas due to photoreactions, since these are always negligible in the system studied here. We also neglect, for simplicity, the sawtooth modulation of the background spectrum due to the IGM line opacity in the Lyman series (Haiman et al. 1997).

\subsection{Initial Conditions}

Within the hierarchical $\Lambda$ CDM model, the first luminous objects are expected to form out of high- $\sigma$ peaks in the random field of primordial density fluctuations. The early (linear) evolution of such a peak, assumed to be an isolated and roughly spherical overdensity, can be described by the top-hat model (see, e.g., Padmanabhan 1993). We use the top-hat approximation to specify the initial DM configuration. In this paper, we investigate the fate of a $2 \sigma$ peak of total mass $10^{8} M_{\odot}$, corresponding to $1.5 \times 10^{7} M_{\odot}$ in baryons. On this mass scale, one can estimate the redshift of collapse (or virialization) to be $z_{\mathrm{vir}} \simeq 10$.
Our simulation is initialized at $z_{i}=100$, by performing the following steps. The collisionless DM particles are placed on a cubical Cartesian grid and are then perturbed according to a given power spectrum $P(k)=A k^{n}$ by applying the Zeldovich (1970) approximation, which also allows us to self-consistently assign initial velocities. The powerlaw index is set to $n=-2.5$, which approximately describes the spectral behavior on the scale $\sim 10^{8} M_{\odot}$. To fix the amplitude $A$, we specify the initial variance of the fluctuation amplitude

$$
\sigma_{i}^{2}=A \sum k^{n} .
$$

The summation is over all contributing modes, where the minimum wavenumber is given by the overall size of the Cartesian box and the maximum wavenumber by the Nyquist frequency. Choosing $\sigma_{i}^{2} \simeq 0.01$, the rms amplitude of fluctuations at the moment of collapse is approximately

$$
\sigma(z=10) \simeq\left(\frac{1+z_{i}}{1+z}\right) \sigma_{i} \simeq 1 .
$$

This choice ensures that the substructure develops on a timescale similar to that of the overall collapse of the background medium. Next, particles within a (proper) radius of $R_{i}=776 \mathrm{pc}$ are selected for the simulation. The resulting number of DM particles is $N_{\mathrm{DM}}=17,074$. Finally, the particles are set into rigid rotation and are endowed with a uniform Hubble expansion (see also Katz 1991). Angular momentum is added by assuming a spin parameter $\lambda=L|E|^{1 / 2} /\left(G M^{5 / 2}\right)=0$ and 0.05 , where $L, E$, and $M$ are the total angular momentum, energy, and mass, respectively. The spin parameter is a measure of the degree of rotational support, such that the ratio of centrifugal to gravitational acceleration is given by $\sim \lambda^{2}$ at virialization. The second value corresponds to the average spin parameter found in cosmological simulations (see, e.g., Barnes \& Efstathiou 1987; Jang-Condell \& Hernquist 2001).

The collisional SPH particles $\left(N_{\mathrm{SPH}}=32,768\right)$ are randomly placed to approximate a uniform initial density. The SPH particles follow the same Hubble expansion and rigid rotation as the DM particles. For the initial gas temperature at $z_{i}=100$, we adopt the value of $200 \mathrm{~K}$ (Tegmark et al. 1997). The fractional free-electron abundance is initialized as $x_{e}=4.6 \times 10^{-4}$ and the hydrogen molecule abundance, in the runs where $\mathrm{H}_{2}$ formation is allowed, as $f_{\mathrm{H}_{2}}=2 \times 10^{-6}$ (Anninos \& Norman 1996).

In Table 1, we summarize the parameters of the large-

\begin{tabular}{|c|c|c|c|c|c|c|}
\hline Run & $\begin{array}{c}M \\
\left(M_{\odot}\right)\end{array}$ & $z_{\mathrm{vir}}$ & $\lambda$ & $\mathrm{H}_{2}$ & LW & $\begin{array}{c}n_{\mathrm{th}} \\
\left(\mathrm{cm}^{-3}\right)\end{array}$ \\
\hline A.................... & $10^{8}$ & 10 & 0 & No & No & $10^{7}$ \\
\hline ............... & $10^{8}$ & 10 & 0.05 & No & No & $10^{7}$ \\
\hline C.... & $10^{8}$ & 10 & 0 & Yes & No & $10^{4}$ \\
\hline $\mathrm{D} \ldots \ldots \ldots \ldots \ldots$ & $10^{8}$ & 10 & 0 & Yes & Yes & $10^{7}$ \\
\hline
\end{tabular}
scale simulations. We discuss the initial state of the refined,

TABLE 1

Parameters for the Different Runs

Note. $-M$ is the total mass of the halo, $z_{\mathrm{vir}}$ is the collapse redshift, $\lambda$ is the spin parameter, $n_{\text {th }}$ is the threshold density for the creation of a sink particle, $\mathrm{H}_{2}$ refers to the absence or presence of molecular hydrogen cooling, and LW denotes whether a photodissociating Lyman-Werner background is included or not. 
small-scale simulation in $\S 4.2$. We emphasize that the initial particle setup, and in particular the realization of the DM fluctuations, is identical for all runs that differ only in the choice of spin, in the presence or absence of a soft UV background, and whether $\mathrm{H}_{2}$ cooling is allowed. The runs with no $\mathrm{H}_{2}$ cooling correspond to the limiting case in which all the $\mathrm{H}_{2}$ has been radiatively destroyed by a soft UV background. In $\S 2$, we have discussed the possible emergence of such an efficient feedback following the buildup of the cosmic UV background close to the epoch of reionization.

\section{THE SIMULATIONS}

In this section, we consider only simulations in which no external UV background is included and defer the discussion of its effect to $\S 5$. We carry out these simulations in two separate steps. First, we simulate the large-scale evolution on the scale of the host halo, $\sim 1 \mathrm{kpc}$, ending with the formation of high-density clumps on a scale of $\sim 1$ pc. Once the mass resolution of the simulation becomes larger than the local Jeans mass, $M_{\mathrm{J}} \lesssim M_{\text {res }}$, a sink particle is created. Consequently, the internal dynamics of a clump cannot be studied any further in these coarse-grain simulations. Second, we follow the evolution of a clump to higher densities and smaller spatial scales, by refining the resolution in the vicinity of the clump to again resolve the Jeans mass. We begin with a discussion of the large-scale evolution.

\subsection{Large-Scale Evolution}

We first describe the run with no spin and with suppressed $\mathrm{H}_{2}$ formation (run A) in more detail and subsequently explore what happens under variations of these assumptions. In Figure 1, we show the initial configuration at $z_{i}=100$, which is identical for all runs. The overdense
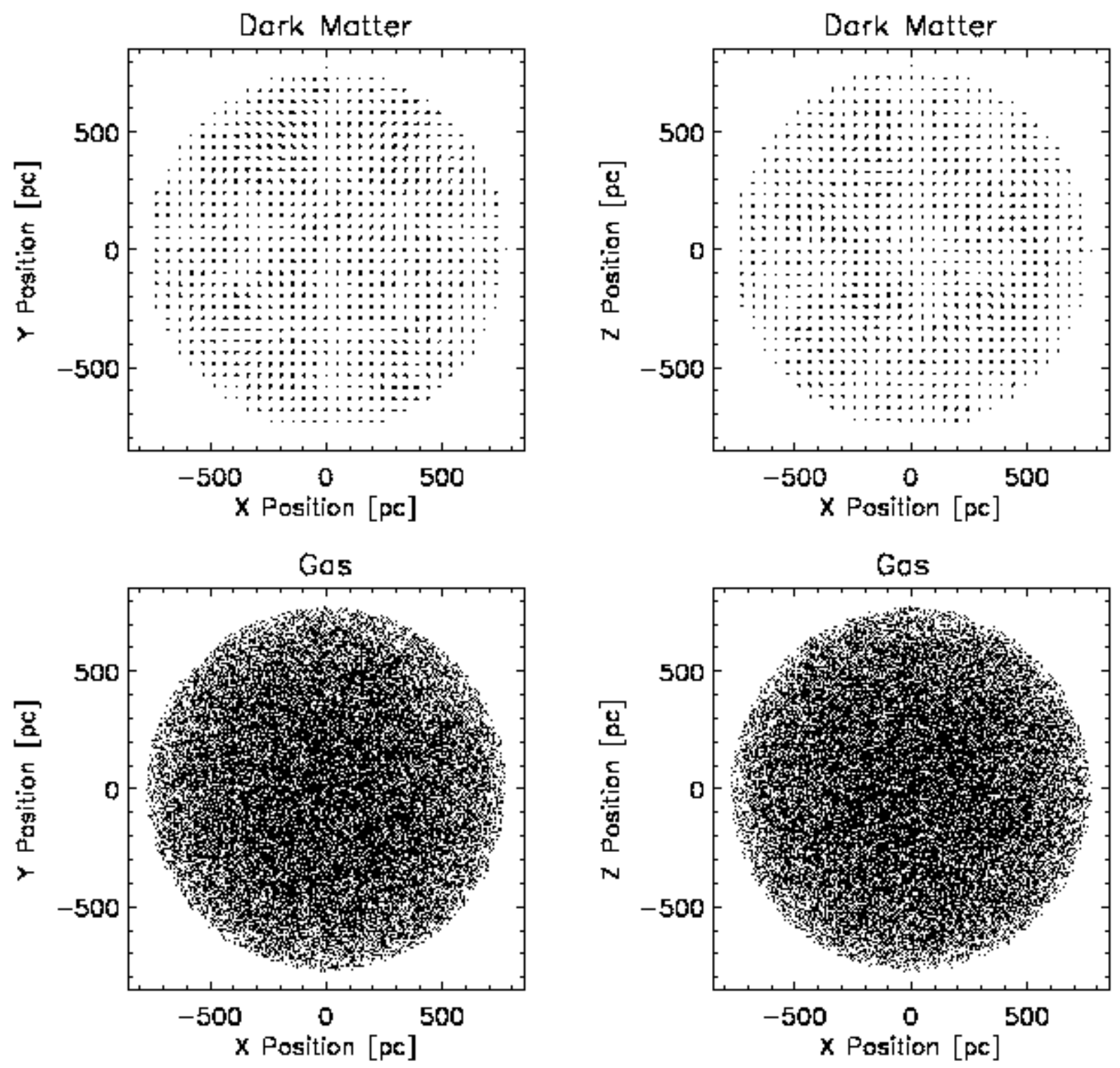

Fig. 1.-Run A: Initial configuration for top-hat collapse at $z_{i}=100$. The halo has a total mass of $10^{8} M_{\odot}$ and is endowed with a Hubble expansion such that virialization occurs at $z_{\mathrm{vir}} \simeq 10$. Top row: DM particles are perturbed from a regular grid according to $P(k) \propto k^{-2.5}$. Bottom row: Gas particles are placed at random and comprise a mass fraction of $15 \%$. Left panels: Face-on view. Right panels: Edge-on view. 
region initially expands with the Hubble flow at a reduced rate, subsequently turns around at $z_{\mathrm{ta}} \sim 16$, and eventually collapses.

Figure 2 shows the system at $z \sim 13$, briefly after turnaround. The DM component has developed a marked substructure in response to the imprinted perturbations, and the baryons have begun to fall into the deepest DM potential wells. Eventually, close to the redshift of virialization, $z_{\mathrm{vir}} \simeq 10$, the DM is undergoing violent relaxation (LyndenBell 1967), resulting in an approximate balance between kinetic and gravitational potential energy.

The baryons, on the other hand, dissipatively settle into the center of the DM halo (see Fig. 3). Focusing on the innermost $\sim 200 \mathrm{pc}$ of the simulation box, Figure 4 displays the central distribution of the gas density. At this stage in the simulation, a high-density sink particle of mass $\sim 3 \times 10^{6} M_{\odot}$ and radius $\lesssim 1 \mathrm{pc}$ has been created. Subsequently, the sink particle grows in mass by continuous accretion from the surrounding diffuse gas.
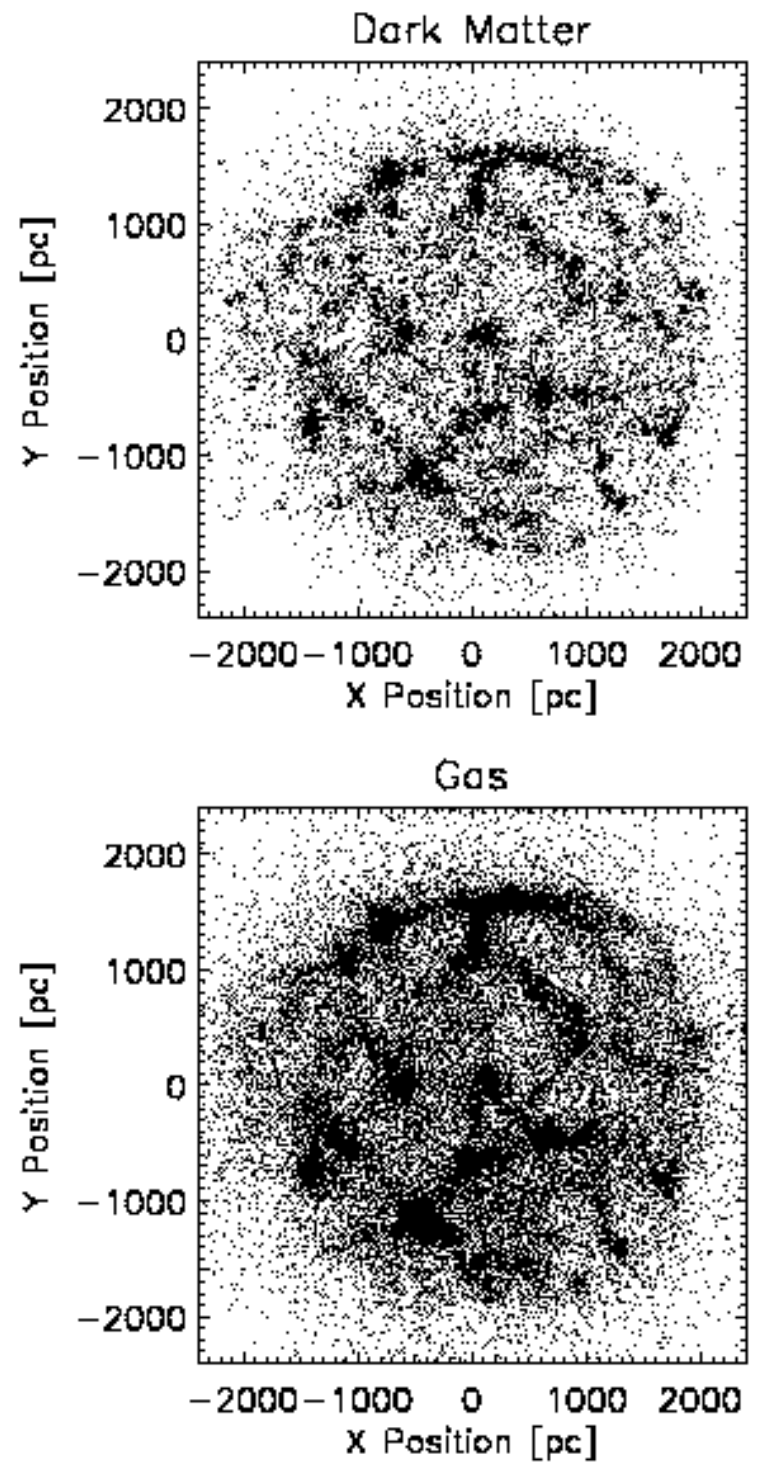

To gain further insight into the physics of the simulation, we consider the thermal and chemical properties of the gas, as illustrated in Figure 5. We show the free-electron abundance, temperature, and Jeans mass as a function of gas density for every SPH particle. This manner of presentation contains an additional dimension of information, concerning the overall timescale of evolution: when the evolution proceeds slowly, particles tend to pile up in the respective plots, whereas only a few particles populate regions in parameter space where the evolution is fast. As can be seen in Figure $5 d$, a gas density of $n \sim 10^{2} \mathrm{~cm}^{-3}$ and a Jeans mass of $M_{\mathrm{J}} \sim 10^{6} M_{\odot}$ approximately mark the transition between phases of slow and fast evolution. Physically, this transition corresponds to the onset of runaway collapse when the clump mass is $M \gtrsim M_{\mathrm{J}}$.

In Figure 6, we show the central gas configuration for the simulation in which the initial spin of the system is nonzero (run B). In this case, a binary system of clumps has formed with a separation of $\sim 10 \mathrm{pc}$. These two clumps individually
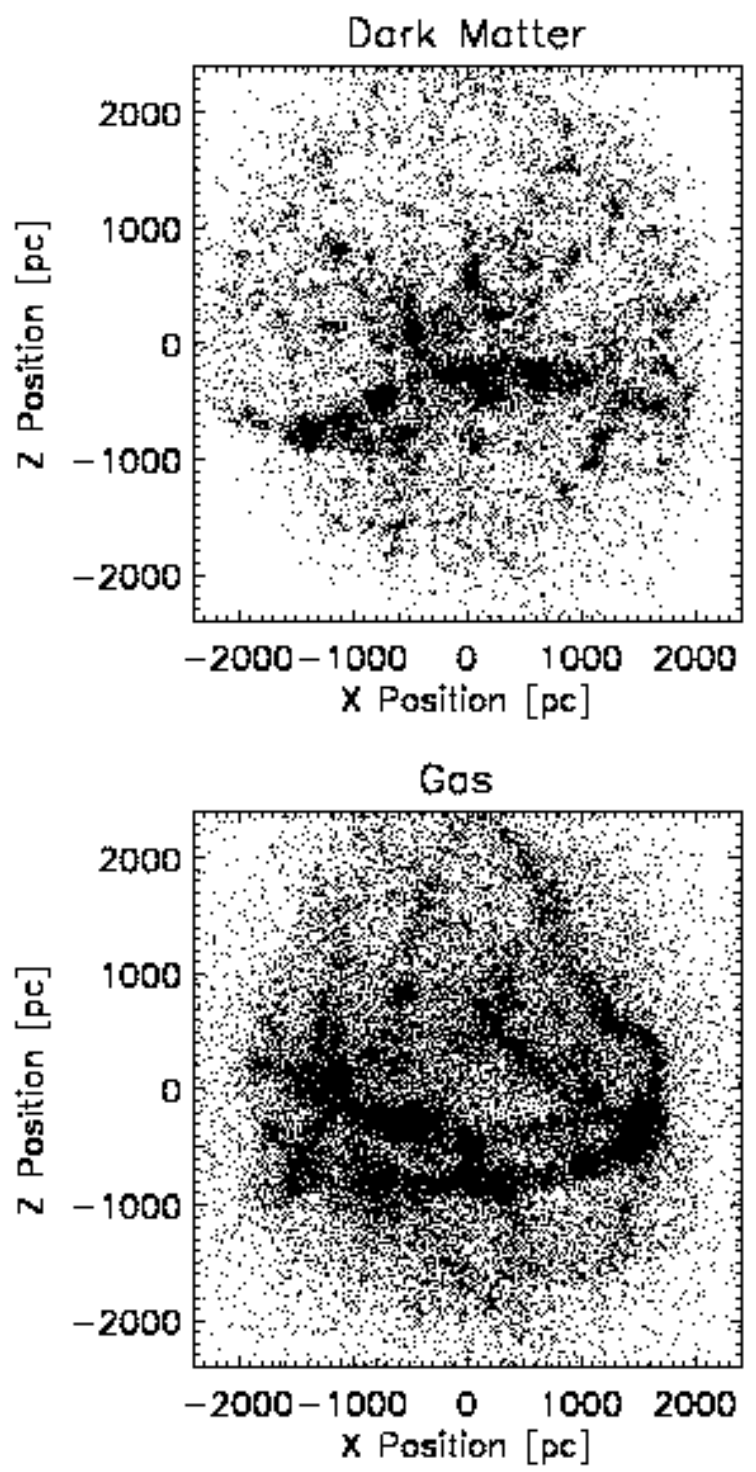

Fig. 2.-Run A: Morphology at $z \sim 13$. The manner of presentation is the same as in Fig. 1, but with a box size of $\sim 5 \mathrm{kpc}$. The DM has developed significant substructure, and the baryons are just beginning to fall into the corresponding potential wells. 

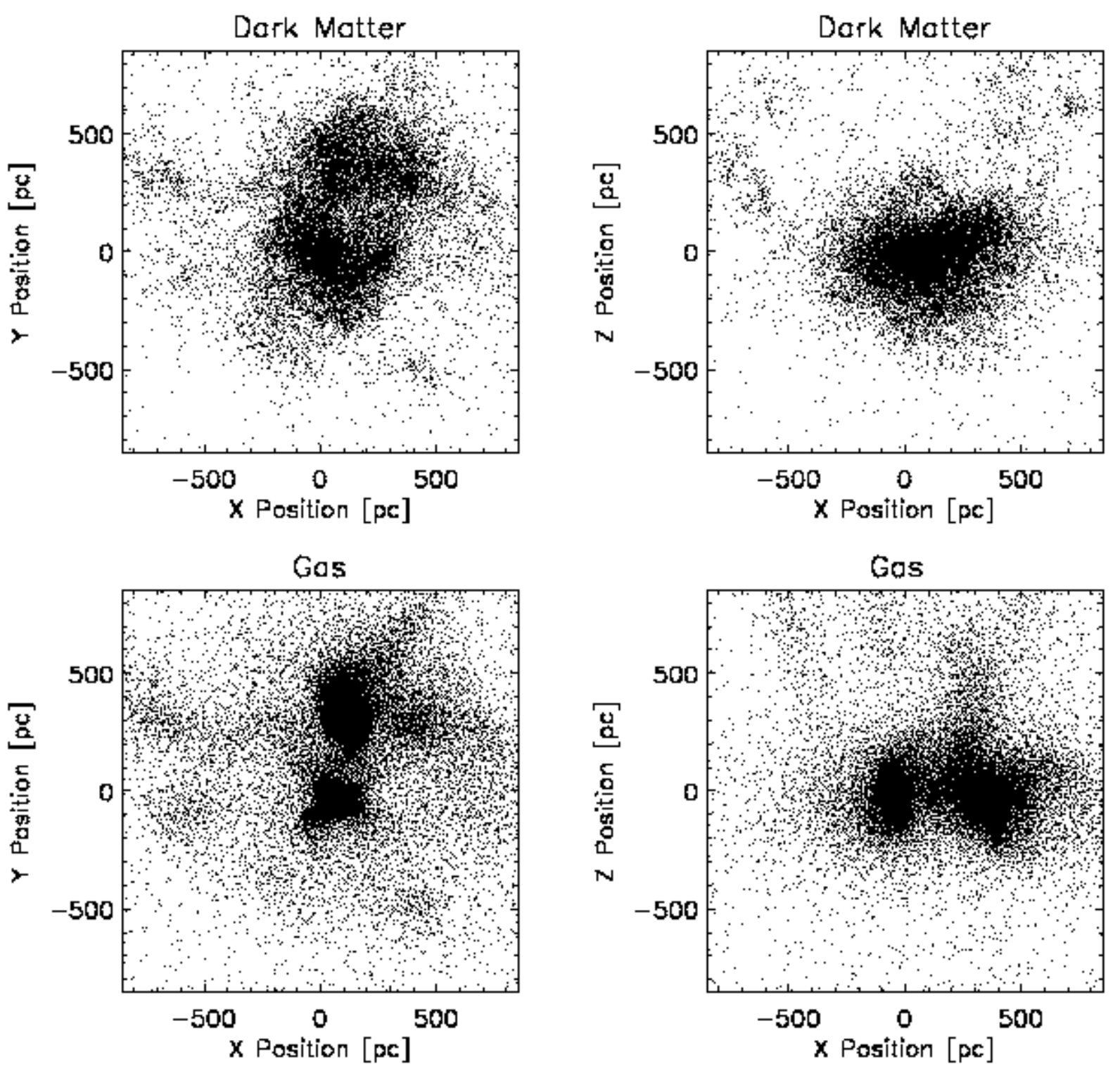

Fig. 3. - Run A: Morphology at $z=10.3$. The convention in Fig. 1 is adopted for the rows and columns. Box size is $\sim 2 \mathrm{kpc}$. The DM is in the process of undergoing violent relaxation with the concurrent smoothing out of substructure. The gas has settled into the center of the DM potential well.

have properties similar to the one clump formed in run A. The binary is rapidly drawn into the center of the halo by dynamical friction, which operates on a timescale $t_{\mathrm{df}} \sim$ $0.1 r^{2} v_{c} /\left(G M_{\mathrm{cl}}\right) \sim 10^{7} \mathrm{yr}$ (Binney \& Tremaine 1987). Here $v_{c}$ is the circular velocity at radius $r$, and $M_{\mathrm{cl}}$ is the clump mass. This timescale is short compared to the Hubble time at $z \sim 10, t_{\mathrm{H}} \simeq 10^{8} \mathrm{yr}$. Such a system of two compact objects is expected to efficiently radiate gravitational waves that could be detected with the planned Laser Interferometer Space Antenna (LISA $;{ }^{5}$ Wyithe \& Loeb 2003b).

Massive clumps, either in isolation or as a binary, are able to form in both runs A and B, which correspond to different values of initial spin. The result that such a compact object can form even in a halo with average initial spin (run B) is seemingly at odds with the earlier investigation by Eisenstein \& Loeb (1995), who have argued that only the

\footnotetext{
${ }^{5}$ See http://lisa.jpl.nasa.gov.
}

lowest spin cosmological perturbations can harbor seed BHs. The gas that is eventually incorporated into the two massive clumps in run B must have been able to efficiently lose angular momentum. It is crucial that the evolution in the $\lambda=0.05$ case leads to the formation of a binary system. Tidal torques can then transfer much of the angular momentum from the gas around each forming clump to the orbital motion of the system, similar to the case of presentday star formation in a clustered environment (see, e.g., Larson 2002; Bate et al. 2003). We plan to more fully address the complex physics of angular momentum transport in virializing DM halos in future work.

This efficient mechanism of assembling a large amount of gas into a compact ( $\lesssim 1 \mathrm{pc}$ ) configuration crucially depends on the suppression of star formation in the collapsing halo. In run $\mathrm{C}$, which otherwise has the same initial conditions as run $\mathrm{A}$, we now allow for the formation of hydrogen molecules. Figure 7 (which should be compared to Fig. 5, run A), shows that the thermal evolution of the gas in this case 


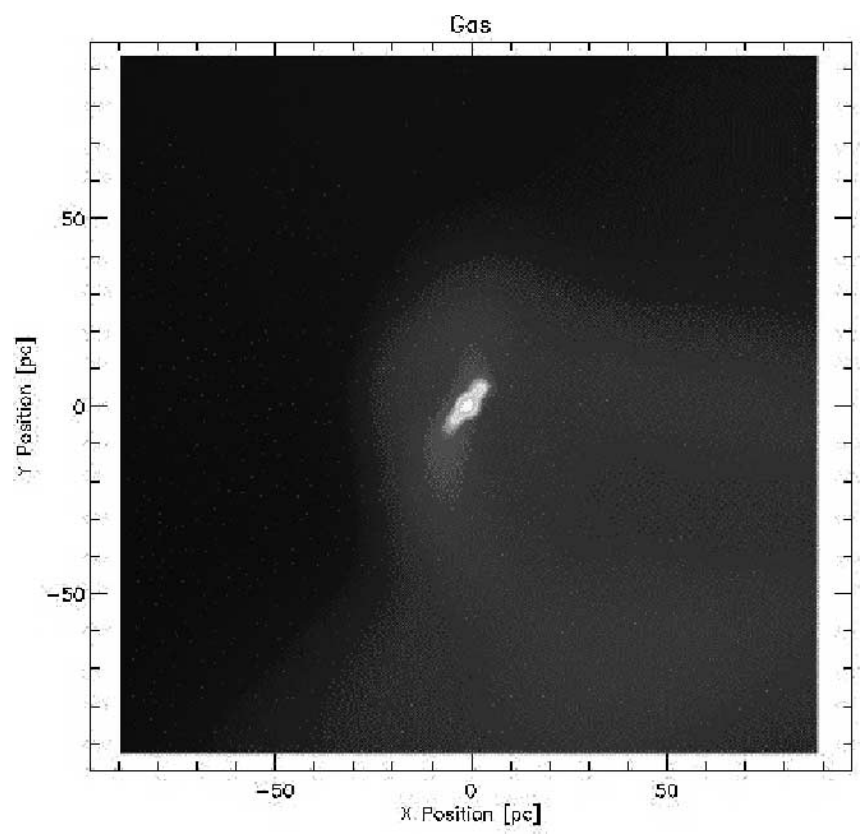

FIG. 4.-Run A: Central gas density at $z=10.3$, in the case with zero initial spin. The box size is $200 \mathrm{pc}$. One compact object has formed in the center with a mass of $2.7 \times 10^{6} M_{\odot}$ and a radius of $\lesssim 1$ pc. [See the electronic edition of the Journal for a color version of this figure.]

proceeds very differently from that in runs A and B. Here the gas is able to cool to temperatures $T \gtrsim 200 \mathrm{~K}$ because of the presence of $\mathrm{H}_{2}$. Provided that $\mathrm{H}_{2}$ formation is not suppressed, the properties of primordial, metal-free gas are rather similar in systems of mass $\sim 10^{8} M_{\odot}$ and in the smaller halos of mass $\sim 10^{6} M_{\odot}$ that are predicted to host the very first stars at $z \simeq 20-30$ (see Bromm et al. 2002, Fig. 10).

The thermal evolution is clearly reflected in the morphology. Figure 8 shows the projected gas density. Because of efficient cooling, the gas can readily fragment and subsequently undergo runaway collapse to form stars already shortly after turnaround. This star formation activity will tend to prevent the assembly of gas in compact clumps in two ways. First, the Population III stars are expected to be short-lived, with lifetimes of $\sim 3 \times 10^{6} \mathrm{yr}$, and they will exert a strong negative feedback on their surroundings upon their deaths as SNe. Second, the SNe will disperse the nucleosynthetic products from the first generation of stars into the remaining gas of the halo. Henceforth, the enriched gas will be able to cool and form stars regardless of whether $\mathrm{H}_{2}$ is present or not. Since the photodissociation of $\mathrm{H}_{2}$ is now no longer able to limit the gas condensation (see $\S 2$ ), the star formation rate is expected to increase substantially (see, e.g., Nishi \& Tashiro 2000; Bromm \& Clarke 2002).

The massive clumps formed in runs A and B are evidently not yet BHs. To examine the subsequent dynamics of the clump, we have resimulated the evolution of a typical clump with higher resolution in order to reach smaller spatial scales. We report on this simulation next.

\subsection{Small-Scale Evolution}

As a representative example, we have selected run A for initializing the refined simulation. We focus on the central region, as shown in Figure 4, and resample the gas within a radius of $\sim 5 \mathrm{pc}$ from the density maximum. The initial moment of the resampled run is chosen to be somewhat earlier than in Figure 4, corresponding to $z \simeq 10.6$, slightly before a sink particle was created in the coarse-grain simulation. In the resampled region, the baryons comprise a mass of $\sim 6 \times 10^{5} M_{\odot}$ and are now the dominant component, compared to only $\sim 10^{5} M_{\odot}$ in dark matter.

After $\sim 10^{4} \mathrm{yr}$, we reach the end state of our refined simulation, at which point the Jeans mass again becomes comparable to the resolution limit. The central gas cloud is in a state of free fall, and we do not see any signs of further subfragmentation. We have verified that fragmentation is not artificially suppressed in the resampling process. To this extent, we have carried out a fiducial simulation that does lead to fragmentation at some point in the evolution and a comparison calculation in which we resample the fluid before fragmentation occurs. We find that the resulting fragmentation pattern is very similar in the two cases, as is physically expected.

The innermost region, of size $\lesssim 0.1 \mathrm{pc}$, comprises $\sim 10^{4}$ $M_{\odot}$ in gas, with densities in excess of $10^{9} \mathrm{~cm}^{-3}$. If we were to continue the simulation further in time, the amount of gas residing inside the central $\sim 0.1$ pc would rapidly increase. Indeed, the mass of the sink particle formed in the largescale simulation of run $\mathrm{A}$, on the order of a few times $10^{6}$ $M_{\odot}$, is indicative of the total baryonic mass that will end up in the central compact object. At this stage, this object is characterized by a ratio $E_{\text {rot }} /\left|E_{\text {grav }}\right| \simeq 0.5$, where $E_{\text {rot }}$ and $E_{\text {grav }}$ are the rotational and gravitational energies of the central compact object, respectively. The angular momentum present in the central clump must have arisen through torques from the clumpy DM distribution during the relaxation process, as run A has zero initial spin. Again, we plan to revisit this issue in our work on angular momentum transport in collapsing DM halos.

The crucial question now is this: What is the further fate of the central object? In particular, do we expect the cloud to fragment and eventually form a central stellar cluster? Or else, will fragmentation be inhibited, as in the earlier, largescale evolution described in the previous section? In the latter case, the additional question arises (Loeb \& Rasio 1994) whether the gas cloud will continue to collapse directly to a black hole or else settle temporarily into a pressuresupported configuration of an SMS (see $\S 17$ in Shapiro \& Teukolsky 1983). We can gain important physical insight into these questions by comparing three important timescales in the problem, namely, the free-fall time, $t_{\mathrm{ff}}$, during which dynamical equilibrium is established, the cooling time, $t_{\text {cool }}$, during which the gas may radiate its thermal energy as it contracts and heats to higher temperatures, and the viscous time, $t_{\mathrm{vis}}$, during which angular momentum is transported. The cooling time remains relatively short and allows continued collapse until the cloud becomes optically thick to the radiation it produces. Spherically symmetric calculations indicate that when the particle density in the contracting gas clump rises above $\sim 10^{17} \mathrm{~cm}^{-3}$, the ionization fraction increases sharply by 7 orders of magnitude to a value $x_{e} \gtrsim 0.1$ (see Omukai 2001, Fig. 4). A clump of mass $\sim 2 \times 10^{6} M_{\odot}$ achieves this dense phase when its radius $R$ shrinks to $\left(R / 10^{16} \mathrm{~cm}\right) \equiv R_{16} \lesssim 0.5$, about 2 orders of magnitude smaller than the value at the end of our simulations. When $R_{16} \lesssim 1$, the optical depth of the cloud to Thomson scattering by free electrons is $\tau \sim 10^{7} R_{16}^{-2}$, and so the cooling 

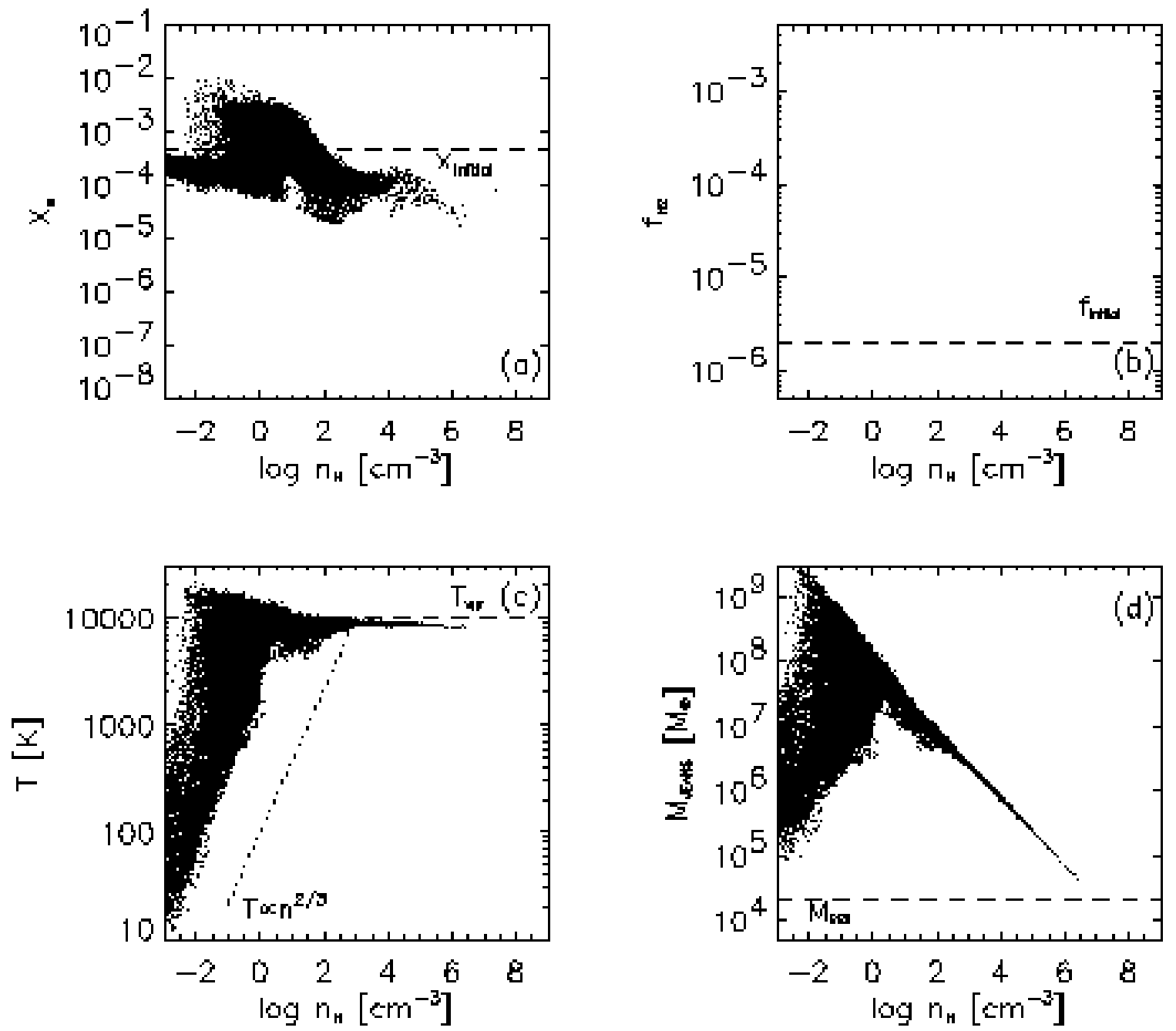

Fig. 5.-Run A: Gas properties at $z=10.3$. (a) Free electron abundance vs. hydrogen number density (in $\mathrm{cm}^{-3}$ ). (b) Hydrogen molecule abundance vs. number density. In this run, we do not allow for the formation of $\mathrm{H}_{2}$ but compare with the corresponding panel in Fig. 7. (c) Gas temperature vs. number density. At densities below $\sim 1 \mathrm{~cm}^{-3}$, the gas temperature rises because of adiabatic compression until it reaches the virial value of $T_{\text {vir }} \simeq 10,000 \mathrm{~K}$. At higher densities, cooling due to atomic $\mathrm{H}$ keeps the gas nearly isothermal. $(d)$ Jeans mass (in $M_{\odot}$ ) vs. number density. The Jeans mass reaches the resolution limit of the simulation, $M_{\text {res }} \simeq 20,000 M_{\odot}$, for hydrogen number densities close to the merging threshold of $n_{\mathrm{th}}=10^{7} \mathrm{~cm}^{-3}$.

time is given by the photon diffusion time $t_{\text {cool }} \sim$ $\tau R / c \sim 10^{5}$ yr $R_{16}^{-1}$. The free-fall time, $t_{\mathrm{ff}} \sim(G \rho)^{-0.5} \sim$ $1 \mathrm{yr} R_{16}^{1.5}$, is much shorter than this cooling time, and so the gas may settle into a radiation pressure-supported configuration resembling a rotating SMS. The viscous time is shorter than the cooling time, $t_{\mathrm{vis}} \lesssim 4 \times 10^{4}$ yr $R_{16}^{0.5} \times$ $\left(P_{\text {gas }} / P_{\text {tot }}\right) \alpha^{-1}$, where $\alpha \lesssim 1$ is a dimensionless measure of the viscosity coefficient (Shakura \& Sunyaev 1973; see $\S 14.5$ in Shapiro \& Teukolsky 1983) in terms of the total (gas+ radiation) pressure, $P_{\text {tot }}=\left(P_{\text {gas }}+P_{\text {rad }}\right) \gg P_{\text {gas }}$. The resulting viscous dissipation will likely heat the optically thick gas close to its virial temperature, since the rotational energy is substantial at the end of our simulation. Combined with additional heating from adiabatic compression, the collapse may be halted as soon as the gas becomes highly optically thick and is unable to cool efficiently. We therefore conclude that if the simulated gas clouds continue to shrink during the early, optically thin (efficient cooling) regime by about 2 orders of magnitude in radius (as they do earlier in the simulations), then they will likely form a rotating SMS.

Recent fully relativistic calculations of the evolution of rotating SMSs have predicted the collapse into a massive BH (Baumgarte \& Shapiro 1999; Shibata \& Shapiro 2002). Under a wide range of initial conditions, a substantial fraction of the mass of the SMS ( $\sim 90 \%)$ is expected to end up in the $\mathrm{BH}$.

\section{EFFECT OF A PHOTODISSOCIATING BACKGROUND}

We now return to the question What is the required level of the $L W$ background to prevent $\mathrm{H}_{2}$ from forming when selfshielding is taken into account? To answer this question, we have carried out a series of simulations in which we allow $\mathrm{H}_{2}$ to form self-consistently in the presence of a soft UV background. We have determined $J_{\text {crit }}$, the critical flux above which $\mathrm{H}_{2}$ is not able to form throughout the 


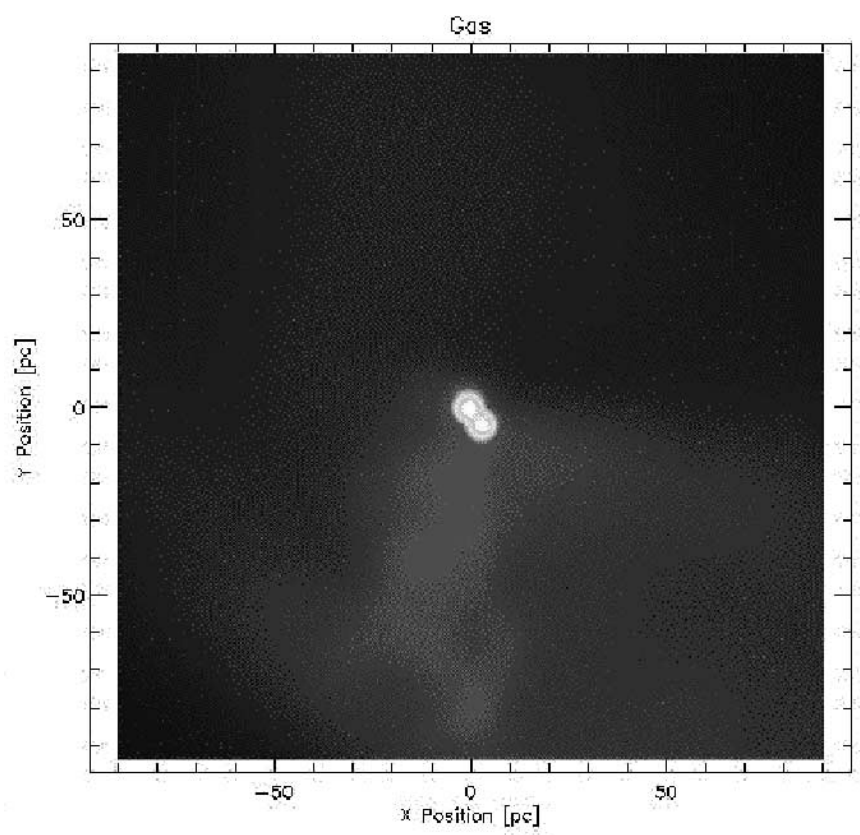

FIG. 6.-Run B: Central gas density at $z \simeq 10$, in the case with an initial spin parameter of $\lambda=0.05$. The box size is $200 \mathrm{pc}$. Here two compact objects have formed in the center with masses of $2.2 \times 10^{6}$ and $3.1 \times 10^{6}$ $M_{\odot}$, respectively, and radii $\lesssim 1 \mathrm{pc}$. [See the electronic edition of the Journal for a color version of this figure.]

simulation volume, for the three spectra described in $\S 3.3$. For both the power-law spectrum and the thermal spectrum with $T_{*}=10^{5} \mathrm{~K}$, we find $J_{\text {crit }} \gtrsim 10^{5}$. This value is larger than the expected background close to reionization (see $\S 2$ ), and it would be difficult to prevent $\mathrm{H}_{2}$ cooling for these two spectra.

A much lower background level, however, suffices in the case of a thermal spectrum with $T_{*}=10^{4} \mathrm{~K}$, for which we find $J_{\text {crit }} \lesssim 10^{3}$, close to the predicted UV background at $z \sim 10$. In Figure 9 we show the timescales that are relevant to understand the abundance of $\mathrm{H}_{2}$ in the simulation with $J_{21}=10^{3}$. It is evident that even when self-shielding is included, the $\mathrm{H}_{2}$ formation time, $t_{\text {form}}$, is longer than the $\mathrm{H}_{2}$ destruction time, $t_{\text {dest }}$, throughout the simulation. Once the gas has reached a density of $n_{\mathrm{H}} \gtrsim 10^{3} \mathrm{~cm}^{-3}$, collisional dissociation becomes very effective in destroying $\mathrm{H}_{2}$ at a gas temperature of $10^{4} \mathrm{~K}$. The ever-increasing column densities and the resulting level of self-shielding, have therefore no effect on the molecule abundance at densities high enough for collisions to become important.

Using an idealized one-zone model to describe the dynamics of a collapsing primordial cloud but otherwise implementing the relevant radiative processes in a sophisticated way, Omukai (2001) has determined the critical UV flux in the case of a Planck spectrum with $T_{*}=10^{4} \mathrm{~K}$ to be $J_{21} \sim 10^{3}$, in good agreement with our numerical simulations.

Both these estimates crucially depend on the presence of a stellar-like radiation background. Therefore, the formation of the first quasars might well require an earlier epoch of star formation. The old puzzle of whether quasars or stars were the first luminous objects in the universe (see, e.g., Silk $\&$ Rees 1998) would then be answered in favor of the latter.

\section{SUMMARY AND CONCLUSIONS}

Our numerical simulations show that metal-free dwarf galaxies at redshifts $z \sim 10$ whose cooling is dominated by atomic transitions (with a virial temperature just above $10^{4}$ $\mathrm{K}$ ) tend to form a central condensation consisting of one or two clumps that contain $\gtrsim 10 \%$ of the total baryonic mass of the galaxy. As long as $\mathrm{H}_{2}$ formation is suppressed, these massive clumps do not fragment but rather cool and continue to collapse isothermally at a temperature of $\sim 10^{4} \mathrm{~K}$. We have simulated the collapse to the point where $\gtrsim 10^{6}$ $M_{\odot}$ of gas condense to a scale $\lesssim 1 \mathrm{pc}$. At the end of our simulation, the clump maintains a nearly free-fall collapse and shows no signs of fragmentation. We expect the collapse to continue until the gas cloud becomes optically thick to Thomson scattering and its cooling time is much longer than the infall time. At this stage the collapse may be halted because of viscous dissipation of rotational energy, and a rotating SMS is likely to form. After shedding a small fraction of its mass, the SMS will collapse to an SMBH (Baumgarte \& Shapiro 1999; Shibata \& Shapiro 2002).

The possible existence of an SMS as an intermediate stage in the formation of the first quasars can be tested by direct observations. The SMS spectrum is expected to be close to thermal at an effective temperature of $\sim 10^{5} \mathrm{~K}$ (Bromm et al. 2001b). The SMS mass can be inferred from its bolometric luminosity, given that the latter equals the Eddington value, $L_{\text {Edd }}=1.4 \times 10^{44} \operatorname{ergs~s}^{-1}\left(M / 10^{6} M_{\odot}\right)$. The emission redshift can be easily derived from the Gunn-Peterson trough produced by Ly $\alpha$ absorption of the neutral IGM (Gunn \& Peterson 1965). Our model predicts that the emission spectrum of the SMS would show only hydrogen and helium lines but no metal lines (such as the broad emission lines observed for quasars at lower redshifts), since metal enrichment would signal small-scale fragmentation into stars and would in turn enable the production of molecules on dust grains. However, all of the above characteristics are common to clusters of Population III stars (Bromm et al. 2001b). To distinguish between an SMS and the extended image of a star cluster requires high angular resolution, possibly achievable with the James Webb Space Telescope. ${ }^{6}$ Alternatively, it is possible to make use of the high probability of gravitational lensing by intervening galaxies out to $z \sim 10$ (Barkana \& Loeb 2000; Wyithe \& Loeb 2002a, 2002b; Comerford, Haiman, \& Schaye 2002). In particular, gravitational microlensing by stars within the lens galaxies (Wyithe \& Loeb 2002b) is only possible for an SMS and not for a star cluster whose extent is much larger than the Einstein radius of a solar-mass star at a cosmological distance, $\sim 10^{-2} \mathrm{pc}$.

Our calculations show that fragmentation into stars is suppressed inside the above dwarf galaxies only if $\mathrm{H}_{2}$ cooling is negligible. The photodissociation of $\mathrm{H}_{2}$ molecules requires a background flux of UV photons below $13.6 \mathrm{eV}$ (to which the neutral IGM is nearly transparent) that could possibly be produced by stars at $z \sim 10$, prior to reionization (see $\S 2$ ).

What is the comoving density of the first generation of massive BHs? At $z \sim 10$, the comoving density of halos with a virial temperature $\gtrsim 10^{4} \mathrm{~K}$ is $\sim 5 \mathrm{Mpc}^{-3}$ (Sheth \& Tormen 1999). Requiring that the total mass density of massive BHs

\footnotetext{
${ }^{6}$ See http://ngst.gsfc.nasa.gov.
} 

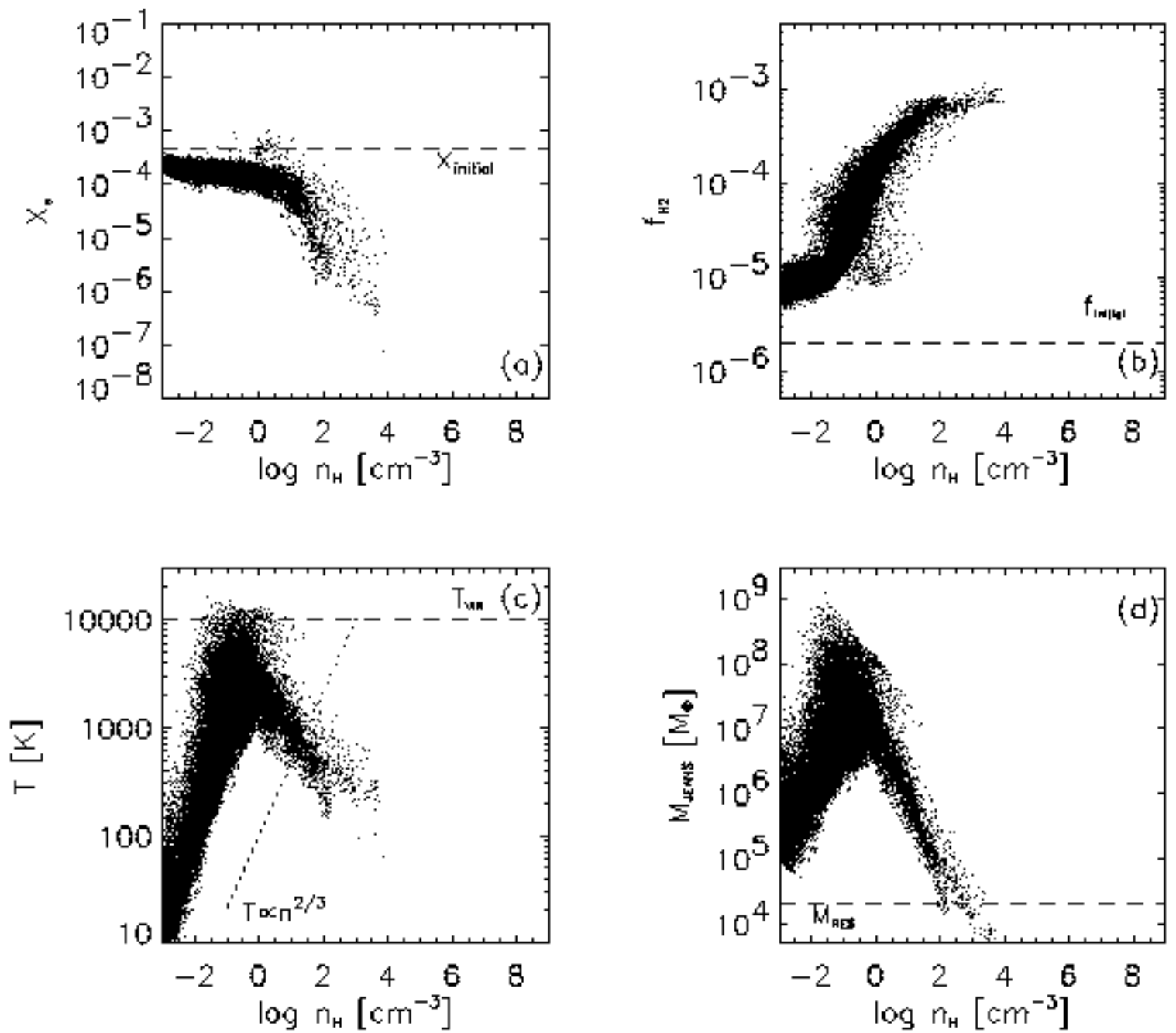

FIG. 7.- Run C: Gas properties at $z \sim 12$. (a) Free electron abundance vs. hydrogen number density (in $\mathrm{cm}^{-3}$ ). At densities exceeding $n_{\mathrm{H}} \sim 10^{3} \mathrm{~cm}^{-3}$, recombination is very efficient, and the gas becomes almost neutral. (b) Hydrogen molecule abundance vs. number density. In this run, we do allow for the formation of $\mathrm{H}_{2}$. The thermal behavior of the gas is markedly different from that in runs A and B, where molecule formation was suppressed. (c) Gas temperature vs. number density. In contrast to runs A and B, cooling due to $\mathrm{H}_{2}$ drives the temperature down at higher density, to values of $\sim 200 \mathrm{~K}$. $(d)$ Jeans mass (in $\left.M_{\odot}\right)$ vs. number density. The Jeans mass reaches the resolution limit of the simulation, $M_{\text {res }} \simeq 20,000 M_{\odot}$, for densities close to the merging threshold of $n_{\text {th }}=10^{4}$ $\mathrm{cm}^{-3}$.

would not exceed the value observed in the local universe (Yu \& Tremaine 2002), 2.5 $\times 10^{5} M_{\odot} \mathrm{Mpc}^{-3}$, we infer that the average $\mathrm{BH}$ mass per halo must be $\lesssim 5 \times 10^{4} M_{\odot}$, much smaller than the characteristic mass of the gas clumps in our simulations. This follows naturally from our expectation that only a small fraction of all halos are both metal-poor and exposed to a high level of UV flux during their entire history (see $\S \S 2$ and 5). Even though a substantial fraction of the IGM volume is not enriched with metals at $z \sim 10$ (Thacker et al. 2002; Furlanetto \& Loeb 2003), most $2 \sigma$ halos may have been enriched by stars that were formed inside their hierarchical building blocks at an earlier cosmic time, when the UV background was much lower. The metals may then facilitate $\mathrm{H}_{2}$ formation on dust particles, as well as efficient cooling through metal emission lines (both atomic and molecular), resulting in further fragmentation. In such a case, only a small minority of all halos forms by direct accretion of pristine gas from the IGM, as we have assumed in our simulations.
Alternatively, the final $\mathrm{BH}$ may contain a small fraction of the initial mass of its parent clump of gas. Since we did not follow the collapse of each clump down to its Schwarzschild radius, we cannot be certain of the final $\mathrm{BH}$ formation efficiency. It is possible that hydrodynamic or radiative feedback from a growing $\mathrm{BH}$ seed at the center limits the final mass that it obtains, so that eventually most of the mass of its parent clump is expelled in a wind because of energy release near the center (see, e.g., Haehnelt, Natarajan, \& Rees 1998; Silk \& Rees 1998). The correlation between BH mass and the velocity dispersion of its host galaxy as observed in the local universe (Merritt \& Ferrarese 2001; Tremaine et al. 2002) would imply an exceedingly low efficiency $\left(\lesssim 10^{-4}\right)$ for the $\mathrm{BH}$ formation process in the dwarf galaxies under consideration (Wyithe \& Loeb 2002c). However, this phenomenological correlation is measured in metal-rich galaxies where molecular cooling and star formation are abundant, and it may not hold under the unusual physical conditions inside the first generation of metal-free 


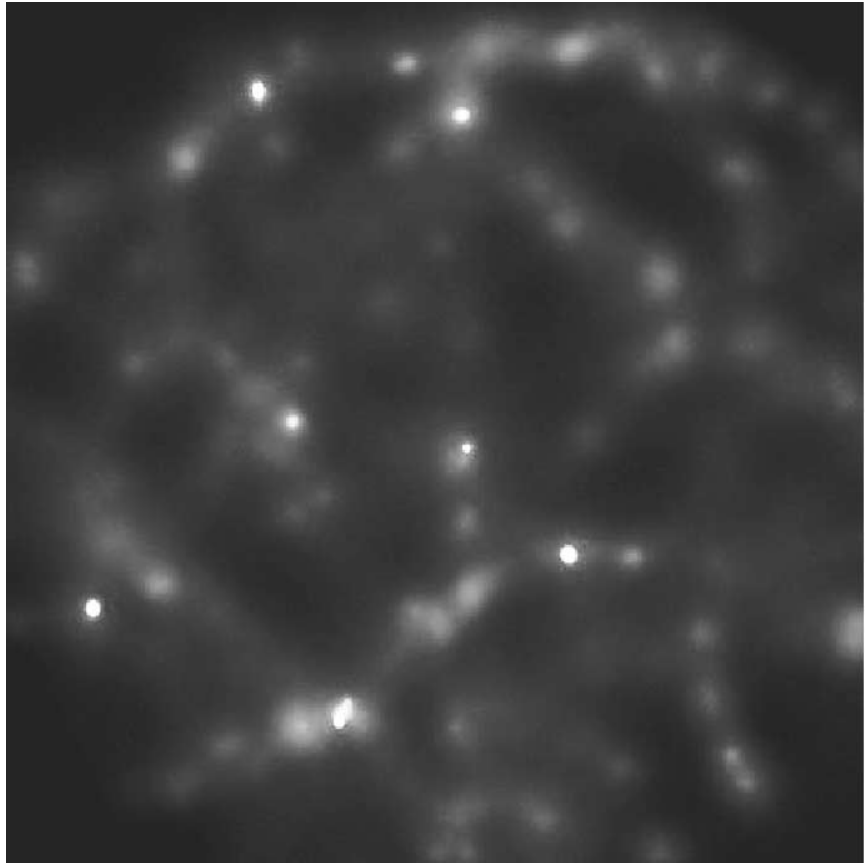

Fig. 8.-Run C: Morphology at $z \sim 12$. Shown is the projected gas density in the $x-y$ plane. The box size is $\sim 3 \mathrm{kpc}$. Here the gas readily fragments and subsequently undergoes runaway collapse to form stars because of the efficient cooling provided by $\mathrm{H}_{2}$. [See the electronic edition of the Journal for a color version of this figure.]

galaxies, where the seeds for the present-day population of BHs were produced.

An early formation of SMBHs would lead to a considerable rate of detectable bursts of gravitational radiation from the coalescence of $\mathrm{BH}$ binaries at high redshifts (Wyithe \& Loeb 2003b). In addition to the commonly discussed origin of binaries in galaxy mergers (a possible example of which has recently been observed in the nearby galaxy NGC 6240 by Komossa et al. 2003), we have demonstrated that $\mathrm{BHs}$ may form in binaries to start with, just as stars do.

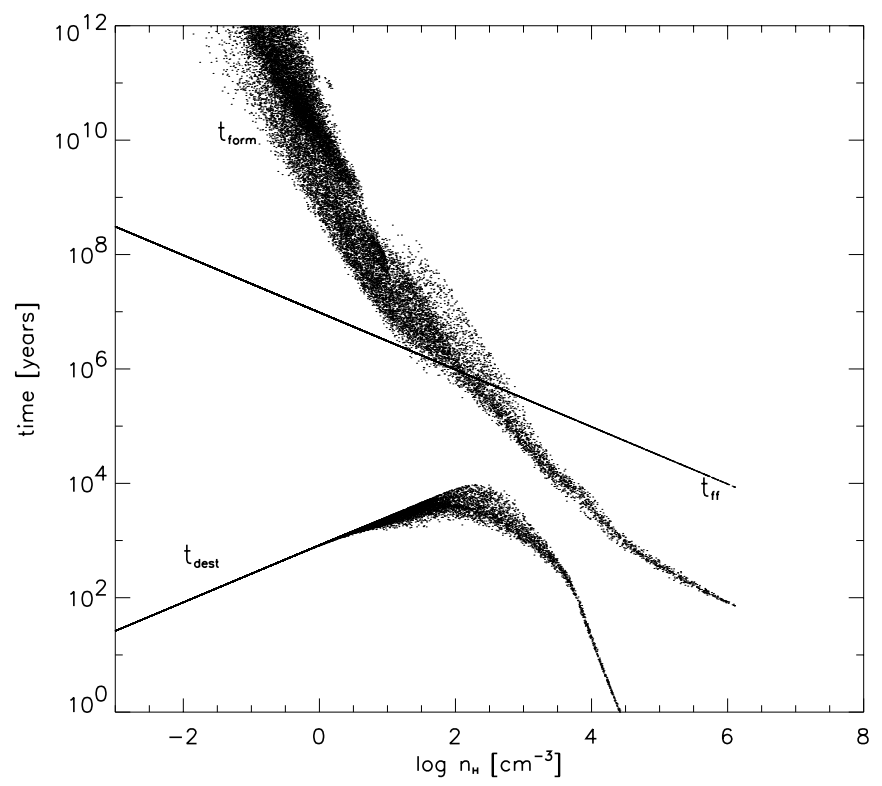

FIG. 9.-Run D: Important timescales at $z=10.6$ for a background stellar spectrum with $T_{*}=10^{4} \mathrm{~K}$. Solid line: Free-fall time vs. hydrogen number density (in $\mathrm{cm}^{-3}$ ). Dots: $\mathrm{H}_{2}$ formation and destruction times vs. $n_{\mathrm{H}}$ for every SPH particle in the simulation. The LW flux is normalized to $J_{21}=10^{3}$ at the Lyman limit. The destruction time increases with density up to $n_{\mathrm{H}} \sim 10^{2} \mathrm{~cm}^{-3}$ because of the effect of self-shielding. $\mathrm{H}_{2}$ is never formed efficiently, however, as $t_{\text {form }}>t_{\text {dest }}$ throughout the simulated volume. At $n_{\mathrm{H}} \gtrsim 10^{3} \mathrm{~cm}^{-3}, \mathrm{H}_{2}$ is readily destroyed via collisions, and $t_{\text {dest }}$ decreases again with density.

We are indebted to Lars Hernquist for making available to us a version of TREESPH. We also thank Rennan Barkana and the anonymous referee for valuable comments on the manuscript. This work has been supported in part by NSF grants AST 00-71019 and AST 02-04514. V. B. thanks the Institute for Advanced Study for its hospitality during the completion of this work. A. L. acknowledges support from the Institute for Advanced Study at Princeton and the John Simon Guggenheim Memorial Fellowship.
Abel, T., Bryan, G. L., \& Norman, M. L. 2000, ApJ, 540, 39 2002, Science, 295, 93

Anninos, P., \& Norman, M. L. 1996, ApJ, 460, 556

Barkana, R., \& Loeb, A. 2000, ApJ, 531, 613

. 2001, Phys. Rep., 349, 125

Barnes, J., \& Efstathiou, G. 1987, ApJ, 319, 575

Bate, M. R., Bonnell, I. A., \& Bromm, V. 2003, MNRAS, 339, 577

Bate, M. R., Bonnell, I. A., \& Price, N. M. 1995, MNRAS, 277, 362

Bate, M. R., \& Burkert, A. 1997, MNRAS, 288, 1060

Baumgarte, T. W., \& Shapiro, S. L. 1999, ApJ, 526, 941

Becker, R. H., et al. 2001, AJ, 122, 2850

Berger, M. J., \& Oliger, J. 1984, J. Comput. Phys., 53, 484

Binney, J., \& Tremaine, S. 1987, Galactic Dynamics (Princeton: Princeton

Univ. Press)

Bromm, V. 2000, Ph.D. thesis, Yale Univ.

Bromm, V., \& Clarke, C. J. 2002, ApJ, 566, L1

Bromm, V., Coppi, P. S., \& Larson, R. B. 1999, ApJ, 527, L5 2002, ApJ, 564, 23

Bromm, V., Ferrara, A., Coppi, P. S., \& Larson, R. B. 2001a, MNRAS, 328,969

Bromm, V., Kudritzki, R. P., \& Loeb, A. 2001b, ApJ, 552, 464

Burles, S., Nollett, K. M., \& Turner, M. S. 2001, ApJ, 552, L1

Cen, R. 1992, ApJS, 78, 341

Ciardi, B., Ferrara, A., Governato, F., \& Jenkins, A. 2000, MNRAS, 314, 611

Comerford, J. M., Haiman, Z., \& Schaye, J. 2002, ApJ, 580, 63

Dekel, A., \& Silk, J. 1986, ApJ, 303, 39

\section{REFERENCES}

Djorgovski, S. G., Castro, S., Stern, D., \& Mahabal, A. A. 2001, ApJ, 560, L5

Draine, B. T., \& Bertoldi, F. 1996, ApJ, 468, 269

Eisenstein, D. J., \& Loeb, A. 1995, ApJ, 443, 11

Fan, X., Narayanan, V. K., Strauss, M. A., White, R. L., Becker, R. H. Pentericci, L., \& Rix, H.-W. 2002, AJ, 123, 1247

Furlanetto, S. R., \& Loeb, A. 2003, ApJ, 588, 18

Galli, D., \& Palla, P. 1998, A\&A, 335, 403

Glover, S. C. O., \& Brand, P. W. J. L. 2001, MNRAS, 321, 385

Gnedin, N. Y., \& Ostriker, J. P. 1997, ApJ, 486, 581

Gunn, J. E., \& Peterson, B. A. 1965, ApJ, 142, 1633

Haehnelt, M. G., Natarajan, P., \& Rees, M. J. 1998, MNRAS, 300, 817

Haiman, Z., Abel, T., \& Rees, M. J. 2000, ApJ, 534, 11

Haiman, Z., \& Loeb, A. 2001, ApJ, 552, 459

Haiman, Z., Rees, M. J., \& Loeb, A. 1997, ApJ, 476, 458 (erratum 484, 985)

Haiman, Z., Thoul, A. A., \& Loeb, A. 1996, ApJ, 464, 523

Hernquist, L., \& Katz, N. 1989, ApJS, 70, 419

Islam, R. R., Taylor, J. E., \& Silk, J. 2003, MNRAS, 340, 647

Jang-Condell, H., \& Hernquist, L. 2001, ApJ, 548, 68

Katz N. 1991, ApJ 368, 325

Kitsionas, S., \& Whitworth, A. P. 2002, MNRAS, 330, 129

Komossa, S., Burwitz, V., Hasinger, G., Predehl, P., Kaastra, J. S., \& Ikebe, Y. 2003, ApJ, 582, L15

Larson, R. B. 2000, in Star Formation from the Small to the Large Scale, ed. F. Favata, A. A. Kaas, \& A. Wilson (ESA SP-445; Noordwijk: ESA), 13 
Loeb, A., \& Rasio, F. A. 1994, ApJ, 432, 52

Lynden-Bell, D. 1967, MNRAS, 136, 101 1969, Nature, 223, 690

Machacek, M. E., Bryan, G. L., \& Abel, T. 2001, ApJ, 548, 509

Mackey, J., Bromm, V., \& Hernquist, L. 2003, ApJ, 586, 1

Madau, P., \& Rees, M. J. 2001, ApJ, 551, L27

Merritt, D., \& Ferrarese, L. 2001, ApJ, 547, 140

Nakamura, F., \& Umemura, M. 2001, ApJ, 548, 19

Nishi, R., \& Tashiro, M. 2000, ApJ, 537, 50

Norman, M. L., \& Bryan, G. L. 1999, in Proc. Int. Conf. on Numerical Astrophysics, ed. S. M. Miyama, K. Tomisaka, \& T. Hanawa (Dordrecht: Kluwer), 19

Oh, S. P., \& Haiman, Z. 2002, ApJ, 569, 558

Omukai, K. 2000, ApJ, 534, 809

$$
\text { 2001, ApJ, 546, } 635
$$

Omukai, K., \& Nishi, R. 1999, ApJ, 518, 64

Padmanabhan, T. 1993, Structure Formation in the Universe (Cambridge: Cambridge Univ. Press)

Press, W. H., Teukolsky, S. A., Vetterling, W. T., \& Flannery, B. P. 1992, Numerical Recipes in Fortran (2d ed.; Cambridge: Cambridge Univ. Press)

Rees, M. J. 1984, ARA\&A, 22, 471

2002, in ESO Astrophys. Symp., Lighthouses of the Universe, ed. M. Gilfanov, R. Sunyaev, \& E. Churazov (Berlin: Springer), 345 Salpeter, E. E. 1964, ApJ, 140, 796
Schneider, R., Ferrara, A., Natarajan, P., \& Omukai, K. 2002, ApJ, 571,30

Shakura, N. I., \& Sunyaev, R. A. 1973, A\&A, 24, 337

Shapiro, S. L., \& Teukolsky, S. A. 1983, Black Holes, White Dwarfs, and Neutron Stars (New York: Wiley)

Sheth, R. K., \& Tormen, G. 1999, MNRAS, 308, 119

Shibata, M., \& Shapiro, S. L. 2002, ApJ, 572, L39

Silk, J., \& Rees, M. J. 1998, A\&A, 331, L1

Stecher, T. P., \& Williams, D. A. 1967, ApJ, 149, L29

Tegmark, M., Silk, J., Rees, M. J., Blanchard, A., Abel, T., \& Palla, F. 1997, ApJ, 474, 1

Thacker, R. J., Scannapieco, E., \& Davis, M. 2002, ApJ, 581, 836

Tremaine, S., et al. 2002, ApJ, 574, 740

Truelove, J. K., Klein, R. I., McKee, C. F., Holliman, J. H., Howell, L. H., Greenough, J. A., \& Woods, D. T. 1998, ApJ, 495, 821

Volonteri, M., Haardt, F., \& Madau, P. 2003, ApJ, 582, 559

Wood, K., \& Loeb, A. 2000, ApJ, 545, 86

Wyithe, J. S. B., \& Loeb, A. 2002a, Nature, 417, 923 2002b, ApJ, 577, 57

2002c, ApJ, 581, 886

$2003 \mathrm{a}$, ApJ, 586, 693 2003b, ApJ, 590,691

Yu, Q., \& Tremaine, S. 2002, MNRAS, 335, 965

Zeldovich, Y. B. 1964, Dokl. Akad. Nauk SSSR, 155, 67

.1970, A\&A, 5, 84 